\title{
Evaluation of Finite-Element Calculations in a Part-Circular Crack by Coherent Optics Techniques
}

\author{
by G.H. Kaufmann, A.M. Lopergolo, S.R. Idelsohn and E.J. Barbero
}

ABSTRACT-Two techniques, speckle photography and holographic interferometry, were used to test three-dimensional finite-element calculations in an internally pressurized cylinder with an external part-circular crack. Opening displacements along the crack line were measured by speckle photography. Radial displacements were obtained from holographic fringe patterns. Good agreement between experimental and numerical data is obtained. Stress-intensity factor variations along the crack front are calculated from numerical results.

\section{Introduction}

Engineering estimates for stress-intensity factors in cracked cylinders under internal pressure have been transformed in a very important problem in fracture mechanics. Failure of nuclear piping systems subjected to a cyclic load condition has been traced to fatigue growth of surface cracks. The stress-intensity factor is required to predict the rate of fatigue crack growth for a crack shape which changes continuously because of its growth. For this reason extensive work has been recently done to calculate stress-intensity factors for various shapes of semi-elliptical and semicircular surface cracks in pressurized cylinders. Three-dimensional numerical analysis using the boundary-integral equation method is reported by Heliot et al. ${ }^{1}$ Atluri and Kathiresan, ${ }^{2}$ Miyazaki et al. ${ }^{3}$ and Raju and Newman ${ }^{4}$ use three-dimensional finite-element methods. Variations of the stress-intensity factor along the crack front have been obtained for a range of crack shapes and sizes for external and internal cracks. In spite of the considerable importance of the experimental evaluation of numerical methods, only a few works are available in the literature.

In the past few years, coherent optics techniques such as holographic interferometry and speckle photography have been used to determine the displacement fields adjacent to the crack tip. ${ }^{5-9}$ Several authors use these measurements to calculate stress-intensity factors for different types of cracks. The use of both techniques in fracture mechanics offers several advantages. As other optical methods, they are noncontact techniques and the model surface needs no preparation. They do not require

G.H. Kaufmann is Professor, and A.M. Lopergolo is Research Fellow, Instituto de Fisica de Rosario (CONICET-UNR), Avda, Pellegrini 250, 2000 Rosario, Argentina. S.R. Idelsohn is Professor, and E.J. Barbero is Research Fellow, Instituto de Desarrollo Tecnológico para la Industria Quimica (CONICET-UNL), P.O. Box 9I, 3000 Santa Fe, Argentina.

Original manuscript submitted: June 26, 1985. Final manuscript received: September 2, 1986. materials with special optical characteristics, so they can be directly applied to any opaque materials such as metals or composites.

The purpose of this paper is to show the usefulness of both techniques in fracture mechanics to evaluate calculations obtained by means of numerical methods. As an example, the case of an external surface part-circular crack in a thick cylinder is analyzed. Finite-element calculations are performed; good agreement is found between experimental and numerical results. Variations of the stress-intensity factor along the crack front calculated by the finite-element idealization are compared with results by Raju and Newman. ${ }^{4}$

\section{Experimental Test}

\section{Specimen}

The circular cylinder used in this study was made of PMMA. This material was chosen because it is a brittle one; so no plastic flow was expected around the crack tip. Hence, experimental results can be compared with those calculated using linear-elastic fracture mechanics. The cylinder had a wall thickness $t$ of $0.5 \mathrm{~cm}$. Its internal radius $R_{i}$ was $2.98 \mathrm{~cm}$. The thickness to radius ratio $t / R_{i}$ was 0.168 . Its length $2 b$ was $20 \mathrm{~cm}$, with a $1-\mathrm{cm}$ clamping on both edges, as shown in Fig. 1.

After the cylinder was cut into size, a central axial partcircular slot was inserted on the external surface of the specimen with a circular saw. The crack length $2 c$ was $1.36 \mathrm{~cm}$. The crack depth $a$ was $0.23 \mathrm{~cm}$, which gave an $a / c$ ratio of 0.338 and an $a / t$ ratio of 0.46 . The slot was $0.02-\mathrm{cm}$ wide and terminated in a vee notch with a very small radius.

Next the specimen was heat treated to relieve stresses. Afterwards, the area surrounding the cracks was sprayed with a thin coat of matt-white paint.

Two square-end steel plates were made to clamp the edges of the cylinder. On each end-plate there was a circular groove, $1-\mathrm{cm}$ deep, which fitted nicely with the cylinder. Before the two ends of the specimen were slid into the groove, a cyanoacrylate cement was poured, thus clamping the ends of the cylinder to the end-plates. Four rods parallel to the cylinder and fastened to both endplates made sure the clamping would not become unglued. Pressure was applied by means of an hydraulic system measured with a manometer. A valve was used to maintain the pressure at a constant value during the exposure time. 
Values of the elastic constant, $E=2920 \mathrm{MPa}$ and $\nu=$ 0.33 , were obtained from a calibration of a bar of the same material using electrical strain gages.

\section{Speckle Photography}

The speckle-photography technique ${ }^{10}$ was used to measure the crack opening (in-plane) displacements $u_{\theta}$ along the slot line. They were obtained by measuring the crack aperture along two lines parallel to the slot direction, one at each side of the crack and at a distance of 0.025 $\mathrm{cm}$ from it. The $u_{\theta}$ displacements were calculated as one half of the difference between the cracks apertures corresponding to a pair of points at each side of the slot.

The sensitivity of this technique is limited by the average speckle size. It can be shown that displacements to be measured must be greater than ${ }^{10}$

$$
\delta_{o}=1.22 \lambda F(1+m) / m
$$

where $\lambda$ is the wavelength of the laser, $F$ is the aperture ratio of the recording optical system and $m$ is its magnification. Nevertheless displacements observed along the crack line were very small (about a few microns) which were outside the sensitivity of the technique given by eq (1). This could be increased, however, by superimposing a small known artificial displacement. For our application, a rigid-body displacement of about three times the speckle diameter was produced by mounting the photographic camera on a vernier table. This displacement was cancelled when the difference between the crack apertures corresponding to a pair of opposite points along the slot was done.

When the cylinder was exposures, its surface move in the radial directon It is well known that out-of-plane displacements produce speckle decorrelation which causes a decrease in fringe contrast and introduces errors in the measurements. ${ }^{10}$ The Rayleigh criterion establishes the movement which can be tolerated without any appreciable degradation of

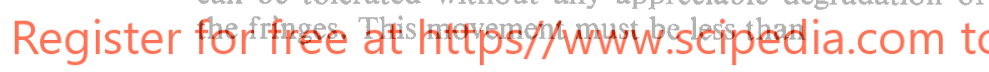

$$
\Delta=4 \lambda F^{2}(1+m)^{2} / m^{2}
$$

For the experiments presented in this paper $\Delta \sim 500 \mu \mathrm{m}$. Taking into account that the radial expansion for the maximum pressure utilized was about $30 \mu \mathrm{m}$, fringes with good contrast were obtained without using any compensation procedure. Figure 2 shows a typical fringe pattern.

To simplify data reduction, the cylinder surface was imaged on to a Kodak SO-253 photographic plate mounted parallel to the specimen by means of a well-corrected camera lens. A $21-\mathrm{cm}$ focal length of $F=8$ aperture was used at $m=2.8$ magnification. The specimen was illuminated by an $\mathrm{He}-\mathrm{Ne}$ laser. Double-exposure specklegrams were analyzed using the pointwise technique. By measuring the spacing and inclination of the fringes, aperture displacements along the crack line were calculated.

\section{Holographic Interferometry}

Holographic interferometry was used to measure the radial (out-of-plane) displacement $u_{r}$ along the crack line. The usual holographic setup to measure out-of-plane displacements was utilized. ${ }^{11}$ The cylinder was normally illuminated with a collimated beam of $\mathrm{He}-\mathrm{N}_{e}$ laser light. It was mounted parallel to the holographic plate. Several double-exposure holograms were recorded on AgfaGevaert 8E75 plates for different pressures. After processing the hologram, the image showing deformation fringes was reconstructed and photographed at normal incidence. Under these conditions, any point on the axial crack line has a radial displacement given by ${ }^{1 !}$

$$
u_{r}=N \lambda / 2
$$

where $N$ takes on integer values at the center of each
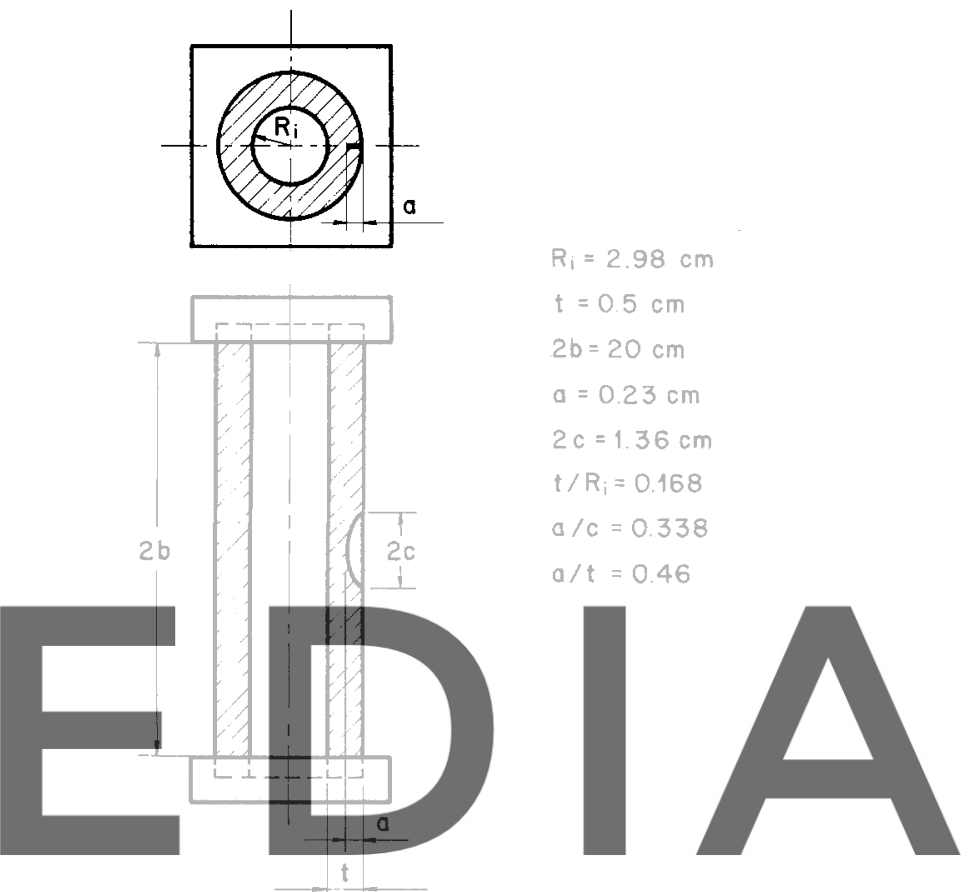

downlorg. id - Circular cylinder with a
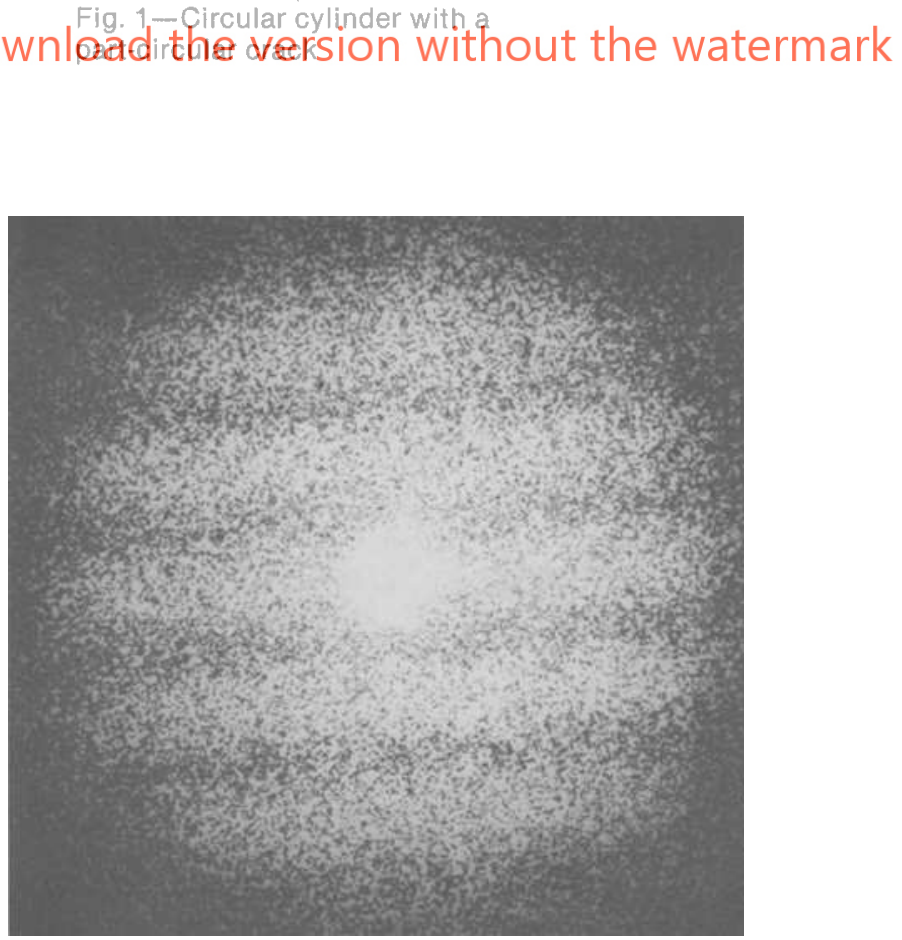

Fig. 2-Typical speckle fringe pattern 